\title{
"C-Methionine Uptake Correlates with Combined 1p and 19q Loss of Heterozygosity in Oligodendroglial Tumors
}

\author{
T. Saito, T. Maruyama, Y. Muragaki, M. Tanaka, M. Nitta, J. Shinoda, T. Aki, H. Iseki, K. Kurisu, and Y. Okada
}

\begin{abstract}
BACKGROUND AND PURPOSE: Oligodendroglial tumors with $1 \mathrm{p} / 19 \mathrm{q} \mathrm{LOH}$ are known to show longer patient survival than those without $1 p / 19 q \mathrm{LOH}$, but the reason for this clinical difference has not been elucidated, to our knowledge. This study was designed to clarify whether uptake of MET correlates with 1p/19q LOH of oligodendroglial tumors.
\end{abstract}

MATERIALS AND METHODS: This study included 102 consecutive patients with supratentorial WHO grade II and III oligodendroglial tumors (39 oligoastrocytic and 63 oligodendroglial tumors) that were resected and diagnosed between January 2008 and August 2011 at Tokyo Women's Medical University Hospital. These patients underwent MET PET T/N ratio measurement before treatment. T/N ratios were calculated by dividing the maximum SUV for the tumor by the mean SUV of the contralateral normal frontal cortex. After surgery, FISH for resected tissues was used to determine 1p/19q LOH.

RESULTS: The mean T/N ratio of tumors with $1 \mathrm{p} / 19 \mathrm{q} \mathrm{LOH}$ was significantly greater than that of tumors without $1 \mathrm{p} / 19 \mathrm{q} \mathrm{LOH}(P=.0166)$. The threshold T/N ratio value of 2.46 was found to correlate significantly with $1 \mathrm{p} / 19 \mathrm{q} \mathrm{LOH}$ by univariate $(P=.0011)$ and multivariate analyses $(P=.0209)$ in all tumors.

CONCLUSIONS: The T/N ratio on MET PET might be a useful aid to the diagnosis of $1 \mathrm{p} / 19 \mathrm{q} \mathrm{LOH}$. Our data add new information on the biology and imaging characteristics of oligodendroglial tumors with $1 \mathrm{p} / 19 \mathrm{q} \mathrm{LOH}$.

ABBREVIATIONS: $\mathrm{FISH}=$ fluorescence in situ hybridization; $L A T 7=$ L-type amino acid transporter; $\mathrm{MET}={ }^{\text {"C }} \mathrm{C}$ methionine; $M I B 1=$ mindbomb homolog $1 ; 1 \mathrm{p} / 19 \mathrm{q}$ $\mathrm{LOH}=$ combined loss of heterozygosity of chromosomes $1 \mathrm{p}$ and 19q; SUV = standard uptake value; $\mathrm{T} / \mathrm{N}=$ tumor/normal; WHO = World Health Organization

$\mathbf{T}$ he $1 \mathrm{p} / 19 \mathrm{q} \mathrm{LOH}$ is present in $50 \%-70 \%$ of oligodendroglial tumors. ${ }^{1}$ Tumors with $1 \mathrm{p} / 19 \mathrm{q}$ LOH show prolonged patient survival compared with those with intact $1 \mathrm{p} / 19 \mathrm{q},{ }^{2,3}$ but the reason for this clinical difference is not well-elucidated. Jenkinson et $\mathrm{al}^{4}$ reported that perfusion MR imaging studies revealed high relative CBV to be more likely in oligodendroglial tumors with $1 \mathrm{p} / 19 \mathrm{q}$ $\mathrm{LOH}$. They speculated that increased vascularity and concomitant drug delivery may contribute to the chemoresponsiveness of

Received February 8, 2012; accepted after revision March 20.

From the Department of Neurosurgery and Faculty of Advanced Techno-Surgery (T.S., T.M., M.T., Y.O.) and Institute of Advanced Biomedical Engineering and Science (Y.M., M.N., H.I.), Tokyo Women's Medical University, Tokyo, Japan; Chubu Medical Center for Prolonged Traumatic Brain Dysfunction (J.S., T.A.), Kizawa Memorial Hospital, Gifu, Japan; and Department of Neurosurgery (K.K.), Graduate School of Biomedical Sciences, Hiroshima University, Hiroshima, Japan.

This study was supported in part by National Cancer Center Research and Development Fund.

The authors have no personal financial or institutional interest in any of the drugs, materials, or devices described in this article.

Please address correspondence to Taiichi Saito, MD, Department of Neurosurgery, Tokyo Women's Medical University, 8-one Kawada-cho, Shinjuku-ku, Tokyo $162-$ 8666, Japan; e-mail: taiichis@gmail.com

http://dx.doi.org/10.3174/ajnr.A3173 such tumors. Preoperative prediction of $1 \mathrm{p} / 19 \mathrm{q}$ LOH by using a noninvasive imaging technique is essential to improve the clinical management of oligodendroglial tumors, such as the surgical strategy. Recently, McGirt et $\mathrm{al}^{5}$ reported that maximum extensive resection for low-grade glioma was associated with longer survival. However, we might be able to avoid doing extensive resection for oligodendroglial tumors with $1 \mathrm{p} / 19 \mathrm{q} \mathrm{LOH}$, which is a good prognostic factor to prevent neurologic deterioration, especially located in the eloquent area.

PET examination of MET uptake is the noninvasive imaging method generally considered to provide the most reliable images for evaluating gliomas and is reported to delineate gliomas more accurately than MR imaging. ${ }^{6}$ Several studies have suggested that MET uptake measured with PET reflects the proliferative index ${ }^{7}$ and microvessel attenuation of glioma tissues, ${ }^{8}$ putative markers of protein synthesis and amino acid transport through microvessels, respectively. Furthermore, Kato et $\mathrm{al}^{7}$ previously showed that MET uptake was significantly higher in oligodendrogliomas than in diffuse astrocytomas; this finding suggests that oligodendrogliomas have a higher vascular bed than diffuse astrocytomas. 
We speculated that MET PET uptake correlated with tumor blood volume, in common with the results of a perfusion MR imaging study, ${ }^{9}$ and may be useful in preoperative prediction of $1 \mathrm{p} / 19 \mathrm{q} \mathrm{LOH}$ in oligodendroglial tumors. To our knowledge, no published study has examined a correlation between MET uptake and $1 \mathrm{p} / 19 \mathrm{q} \mathrm{LOH}$ for patients with oligodendroglial tumors. The aim of this study was to determine whether MET uptake on a preoperative PET study correlates with $1 \mathrm{p} / 19 \mathrm{q} \mathrm{LOH}$ of oligodendroglial tumors.

\section{MATERIALS AND METHODS \\ Patients and Histologic Diagnosis}

This study included 102 consecutive patients with WHO grade II and III supratentorial oligodendroglial tumors that were resected and diagnosed between January 2008 and August 2011 at Tokyo Women's Medical University Hospital. The histopathologic assessment revealed 63 oligodendroglial tumors (41 oligodendrogliomas and 22 anaplastic oligodendrogliomas) and 39 oligoastrocytic tumors (22 oligoastrocytomas and 17 anaplastic oligoastrocytomas).

The study protocol was approved by the institutional review board of our institution; written informed consent from the patients was waived. To protect patient privacy, we removed all identifiers from our records on the completion of our analyses.

\section{PET}

PET scans were obtained as previously described. ${ }^{7}$ The PET study was performed according to the standardized procedure used in Chubu Medical Center. The PET scanner was an Advance NXi Imaging System (GE Yokokawa Medical Systems, Tokyo, Japan), which provides 35 transaxial images at $4.25-\mathrm{mm}$ intervals. The in-plane spatial resolution (full width at half maximum) was 4.8 $\mathrm{mm}$, and the scan mode was the standard 2D mode. Before the emission scan was obtained, a 3-minute transmission scan was obtained to correct photon attenuation with a ring source containing ${ }^{68} \mathrm{Ge}$. A dose of $7.0 \mathrm{MBq} / \mathrm{kg}$ of MET was injected intravenously. The emission scan was acquired for 30 minutes, beginning 5 minutes after the MET injection. The images were reconstructed with the ordered-subsets expectation maximization algorithm.

\section{MR Imaging}

MR imaging was performed on a $1.5 \mathrm{~T}$ system (Signa; GE Healthcare, Milwaukee, Wisconsin). T1- and T2-weighted images were acquired by using our standard protocol. For coregistration of metabolic and anatomic data, we also acquired 3D spoiled gradient-echo images after administration of $0.2 \mathrm{~mL} / \mathrm{kg}$ of gadopentetate dimeglumine (Magnevist; Nihon Shering, Osaka, Japan) by using the following parameters: no gap; 1.0 -mm thickness; TR, 20 ms; TE, $1.6 \mathrm{~ms}$; flip angle, $15^{\circ}$; NEX, 1; and axial views.

\section{Data Analysis}

Data analysis was performed as previously reported. ${ }^{7}$ Tracer accumulation in the region of interest was analyzed as the SUV, which is the activity concentration in the region of interest at a fixed time point divided by the injected dose normalized to the patient's measured weight. The MET T/N ratios were calculated by dividing the maximum SUV for the tumor by the mean SUV of the contralateral normal frontal cortex. The tumor maximum SUVs were selected showing the highest accumulation, and a circular reference region of interest was drawn with a diameter of 10 $\mathrm{mm}$ on each of the 3 axial planes. Coregistration of PET and MR imaging was undertaken in all cases with Dr. View, an imageanalysis software package (AJS, Tokyo, Japan). If increased accumulation was absent or not clear, a region of interest was selected in consultation with the fusion image. We used the $\mathrm{T} / \mathrm{N}$ ratio instead of absolute SUV because of the high unexplained intersubject variability of the SUV. We used the tumor maximum SUV instead of the tumor mean SUV to minimize the effect of tumor heterogeneity. For evaluation of the diagnostic efficacy of the T/N ratio in discrimination of $1 \mathrm{p} / 19 \mathrm{LOH}$-positive and -negative tumors, receiver operating characteristic curve analysis was performed with calculation of the area under the curve. For this purpose, the Youden index was used to calculate the optimal cutoff value of the $\mathrm{T} / \mathrm{N}$ ratio. ${ }^{10}$ Consequently, we selected a $\mathrm{T} / \mathrm{N}$ ratio value of 2.46 as the cutoff point and classified the patients into 2 groups: those with tumors with $\mathrm{T} / \mathrm{N}<2.46$ and $\mathrm{T} / \mathrm{N} \geq 2.46$.

\section{Tissue Specimens and Immunohistochemical Staining}

Tumors were classified into histologic subtypes from representative hematoxylin-eosin slides according to the WHO criteria. An avidin-biotin immunoperoxidase or Simple Stain MAX-peroxidase (Nichirei, Tokyo, Japan) technique was used to perform an MIB1 monoclonal antibody (DAKO, Glostrup, Denmark) assay according to the manufacturer's instructions in selected sections of each case. We determined the MIB1 labeling index by counting 1000 tumor cell nuclei. We selected the mean value of the MIB1 labeling index as the cutoff point and classified the patients into 2 groups: those with $<7.5 \%$ and $\geq 7.5 \%$.

\section{Molecular Studies (Loss of Heterozygosity of Chromosomes Ip and 19q)}

The standard method of FISH was used to determine the $1 \mathrm{p} / 19 \mathrm{q}$ deletion as described previously. ${ }^{11}$ The 2-color FISH assay was performed on 5-mm-thick sections, by using mixed 1p36/1q25 and $19 q 13 / 19$ p13 dual-color probe sets (catalog no. 30-231003; Abbott Molecular, Abbott Park, Illinois).

Samples showing sufficient FISH efficiency (90\% nuclei with signals) were evaluated. Signals were scored in at least 200 nonoverlapping intact nuclei. Deletions of $1 \mathrm{p}$ and $19 \mathrm{q}$ were defined as $33 \%$ of tumor nuclei containing the LOH pattern (or the signal ratio of $1 \mathrm{p} / 1 \mathrm{q}<0.84$, and $19 \mathrm{q} / 19 \mathrm{p}<0.85$ ), as reported by van den Bent et al. ${ }^{12}$

\section{Statistical Analysis}

Data were analyzed by using the Statistical Package for the Social Sciences (Version 16.0) software package (SPSS, Chicago, Illinois). Correlations between the status of $1 \mathrm{p} / 19 \mathrm{q} \mathrm{LOH}$ and patient characteristics (sex, age, calcification on CT images, MIB1 labeling index, and T/N ratio) were calculated by using the $\chi^{2}$ test. A $t$ test was used for statistical comparison of the $\mathrm{T} / \mathrm{N}$ ratio between grades II and III in all tumors, 1p/19q LOH-positive and $1 \mathrm{p} / 19 \mathrm{q}$ $\mathrm{LOH}-$ negative in all tumors, within grade II tumors, and within grade III tumors.

Multivariate logistic regression analysis was used to explore 
Table 1: Patient demographics, clinical data, pathologic findings, and $\mathrm{CT}$ findings

\begin{tabular}{lccc}
\hline \multicolumn{1}{c}{ Variable } & $\begin{array}{c}\text { WHO } \\
\text { Grade II }\end{array}$ & $\begin{array}{c}\text { WHO } \\
\text { Grade III }\end{array}$ & Total \\
\hline No. of cases & 63 & 39 & 102 \\
Sex (M/F) & $32: 31$ & $23: 16$ & $55: 47$ \\
Mean age (range) (yr) & $40.9(19-65)$ & $40.2(19-69)$ & $40.7(19-69)$ \\
Mean resection rate & $85.1 \%$ & $90.7 \%$ & $87.3 \%$ \\
Histology & & & \\
$\quad$ Oligodendroglioma & 41 & 22 & 63 \\
$\quad$ Oligoastrocytoma & 22 & 17 & 39 \\
1p/19q LOH & & & \\
Yes & $47 / 63(75 \%)$ & $26 / 39(67 \%)$ & $73 / 102(72 \%)$ \\
$\quad$ No & $16 / 63(25 \%)$ & $13 / 39(33 \%)$ & $29 / 102(28 \%)$ \\
MIB7 labeling index & $4.29 \pm 2.06$ & $12.64 \pm 8.53$ & $7.48 \pm 6.82$ \\
$\quad$ (mean) (\%) & & & \\
Calcification (head CT) & & & \\
Yes & $25 / 63(40 \%)$ & $12 / 39(31 \%)$ & $37 / 102(36 \%)$ \\
$\quad$ No & $38 / 63(60 \%)$ & $27 / 39(69 \%)$ & $65 / 102(64 \%)$ \\
\hline
\end{tabular}

the relationships between the status of $1 \mathrm{p} / 19 \mathrm{q} \mathrm{LOH}$ and variables such as sex, age, calcification, and T/N ratio. A $P$ value $<.05$ indicated a statistically significant difference.

\section{RESULTS}

The demographics of the patient population, histologic diagnosis, status of the $1 \mathrm{p} / 19 \mathrm{q}$, MIB1 labeling index, and CT findings are shown in Table 1 . The mean $\mathrm{T} / \mathrm{N}$ ratio was significantly higher for grade III tumors $(3.323 \pm 1.386)$ than for grade II tumors $(2.674 \pm 1.003)($ Fig $1 A)$. Furthermore, the mean $\mathrm{T} / \mathrm{N}$ ratio of tumors with $1 \mathrm{p} / 19 \mathrm{q}$ LOH $(3.100 \pm 1.163)$ was significantly greater than that of tumors without $1 \mathrm{p} / 19 \mathrm{q}$ LOH $(2.473 \pm 1.193)$ in all tumors (Fig $1 B$ ). Within grade II tumors, the mean $\mathrm{T} / \mathrm{N}$ ratio
Table 2: Subgroups of 1p/19q LOH status and patient characteristics $^{\mathrm{a}}$

\begin{tabular}{lccc}
\hline & $\begin{array}{c}1 \mathrm{p} / 19 q \\
\text { LOH-Positive }\end{array}$ & $\begin{array}{c}1 \mathrm{p} / 19 q \\
\text { LOH-Negative }\end{array}$ & $\begin{array}{c}\boldsymbol{P} \\
\text { Value }\end{array}$ \\
\hline $\begin{array}{l}\text { No. of cases } \\
\text { Sex }\end{array}$ & 73 & 29 & \\
$\quad$ Male & 37 & 18 & .2981 \\
$\quad$ Female & 36 & 11 & \\
Age (mean) & & & \\
$\quad<41$ yr & 38 & 21 & .0603 \\
$\quad \geq 41$ yr & 35 & 8 & \\
Calcification & & & \\
$\quad$ Yes & 28 & 2 & $.0037^{\mathrm{b}}$ \\
$\quad$ No & 45 & 27 & \\
MIB1 labeling index (mean) & & & \\
$\quad<7.5 \%$ & 50 & 19 & .7727 \\
$\quad \geq 7.5 \%$ & 23 & 10 & \\
T/N ratio & & & \\
$\quad<2.46$ & 22 & 19 & $.0011^{\mathrm{b}}$ \\
$\quad \geq 2.46$ & 51 & 10 & \\
\hline
\end{tabular}

a Statistical analysis was performed by using the $\chi^{2}$ test

${ }^{\mathrm{b}} \mathrm{P}<.05$.

of tumors with $1 \mathrm{p} / 19 \mathrm{q} \mathrm{LOH}(2.921 \pm 1.013)$ was also significantly higher than that of tumors without $1 \mathrm{p} / 19 \mathrm{q} \mathrm{LOH}(1.946 \pm 0.501)$ (Fig 1C). However, no significant difference was observed in the mean $\mathrm{T} / \mathrm{N}$ ratio between tumors with $(3.423 \pm 1.357)$ and without $(3.122 \pm 1.478) 1 \mathrm{p} / 19 \mathrm{q} \mathrm{LOH}$ within grade III tumors (Fig 1D).

Univariate analyses showed no significant correlation between 1p/19q LOH status and sex, age, or MIB1 labeling index (Table 2), but $1 \mathrm{p} / 19 \mathrm{q}$ LOH status significantly correlated with the presence of calcification and the threshold $\mathrm{T} / \mathrm{N}$ ratio value of 2.46 .

Multivariate analyses by using the logistic regression model showed that only the thresh-

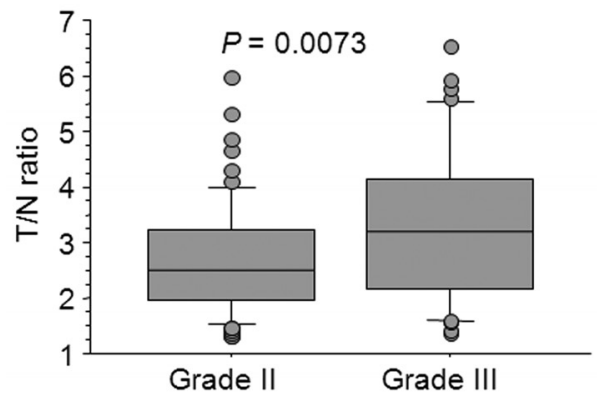

A

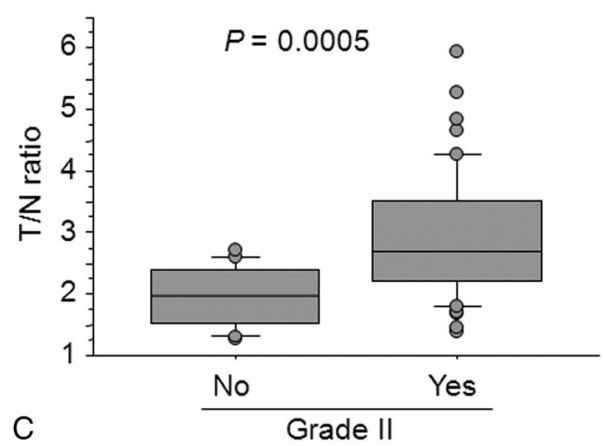

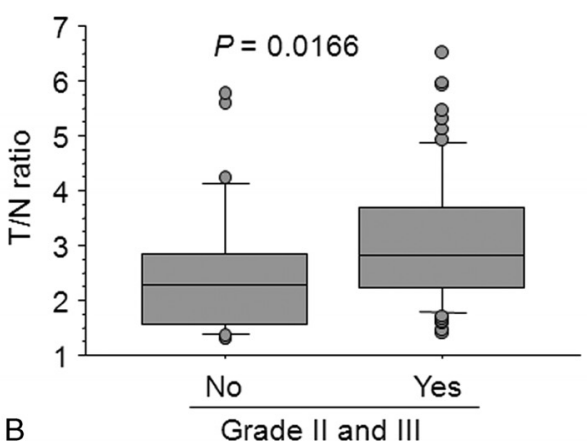

B

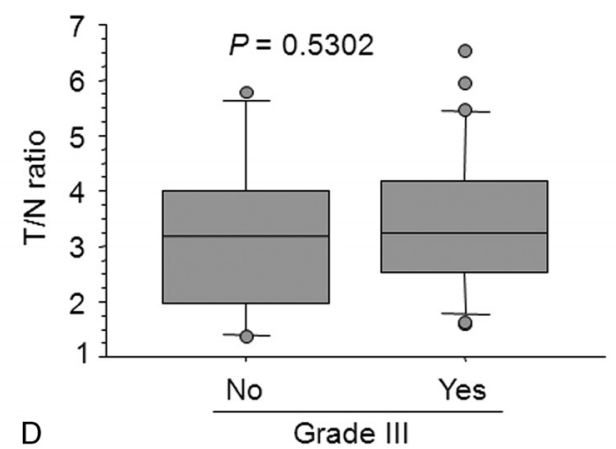

FIG 1. A, Boxplots of $\mathrm{T} / \mathrm{N}$ ratio values for grade II and III tumors. B, Boxplots of $\mathrm{T} / \mathrm{N}$ ratio values for $\mathrm{lp} / 19 \mathrm{q}$ $\mathrm{LOH}$-positive and -negative tumors in all tumors. C, Boxplots of $\mathrm{T} / \mathrm{N}$ ratio values for $\mathrm{lp} / 19 \mathrm{q} \mathrm{LOH}$-positive and -negative in grade II tumors. $D$, Boxplots of $\mathrm{T} / \mathrm{N}$ ratio values for $1 \mathrm{p} / 19 \mathrm{q} \mathrm{LOH}$-positive and -negative in grade III tumors. Yes indicates $1 \mathrm{p} / 19 \mathrm{q} \mathrm{LOH}$-positive; No, 1p/19q LOH-negative. old $\mathrm{T} / \mathrm{N}$ ratio value of 2.46 significantly correlated with the presence of $1 \mathrm{p} / 19 \mathrm{q} \mathrm{LOH}$ in all tumors (Table 3) and also within grade II tumors (Table 3). No significant correlation was observed between $1 \mathrm{p} / 19 \mathrm{q} \mathrm{LOH}$ and the threshold $\mathrm{T} / \mathrm{N}$ ratio value of 2.46 within grade III tumors (Table 3). Representative images are shown in Figs 2 and 3 .

\section{DISCUSSION}

We have demonstrated by PET that $1 \mathrm{p} / 19 \mathrm{q}$ LOH correlates with MET uptake in oligodendroglial tumors. The threshold $\mathrm{T} / \mathrm{N}$ ratio value of 2.46 might be a useful aid to differentiate $1 \mathrm{p} / 19 \mathrm{q}$ LOHpositive from -negative tumors. Several studies have aimed at preoperatively predicting $1 \mathrm{p} / 19 \mathrm{q}$ LOH by using 
Table 3: Multivariate logistic regression model predicting 1p/19q LOH

\begin{tabular}{|c|c|c|c|}
\hline Regressor & Coefficient & OR $(95 \% \mathrm{Cl})$ & $\begin{array}{c}P \\
\text { Value }\end{array}$ \\
\hline \multicolumn{4}{|l|}{ All tumors } \\
\hline Sex (female vs male) & 1.193 & $0.533(0.190-1.498)$ & .2330 \\
\hline Age ( $<41$ yr vs $>41 \mathrm{yr}$ ) & 1.195 & $1.853(0.674-5.094)$ & .1690 \\
\hline Calcification (yes vs no) & 1.785 & $4.284(0.867-21.172)$ & .0743 \\
\hline $\mathrm{T} / \mathrm{N}$ ratio $(<2.46$ vs $\geq 2.46$ ) & 2.309 & 3.449 (1.206-9.868) & $.0209^{b}$ \\
\hline \multicolumn{4}{|l|}{ Grade II tumors } \\
\hline Sex (female vs male) & 0.962 & $0.507(0.127-2.025)$ & .3362 \\
\hline Age $(<41$ yr vs $>41$ yr) & 0.512 & $1.430(0.364-5.616)$ & .6085 \\
\hline Calcification (yes vs no) & 1.086 & $3.513(0.364-33.917)$ & .2773 \\
\hline $\mathrm{T} / \mathrm{N}$ ratio $(<2.46$ vs $\geq 2.46$ ) & 2.546 & 7.522 (1.591-35.569) & $.0109^{b}$ \\
\hline \multicolumn{4}{|l|}{ Grade III tumors } \\
\hline Sex (female vs male) & 0.821 & $0.516(0.107-2.503)$ & .4117 \\
\hline Age $(<41$ yr vs $>41$ yr) & 1.060 & $2.398(0.476-12.083)$ & .2892 \\
\hline Calcification (yes vs no) & 1.616 & 6.839 (0.664-70.451) & .1061 \\
\hline $\mathrm{T} / \mathrm{N}$ ratio $(<2.46$ vs $\geq 2.46)$ & 0.683 & $1.762(0.347-8.953)$ & .4945 \\
\hline
\end{tabular}

Note:-OR indicates odds ratio; $\mathrm{Cl}$, confidence interval.

a Statistical analysis was performed using a logistic regression model. ${ }^{\mathrm{b}} \mathrm{P}<.05$.

a noninvasive technique. Jenkinson et $\mathrm{al}^{13}$ reported that tumors with sharp smooth imaging features and homogeneous signal intensity on MR imaging were more likely to have intact 1p/19q.
Furthermore, Brown et $\mathrm{al}^{14}$ showed that the S-transform-based quantitative MR imaging texture of T2 sequences may have the potential to recognize $1 \mathrm{p} / 19 \mathrm{q} \mathrm{LOH}$ with a sensitivity of $93 \%$ and a specificity of $96 \%$. Walker et $\mathrm{al}^{15}$ used PET and single-photon emission CT studies to show that oligodendroglial tumors with $1 \mathrm{p} / 19 \mathrm{q}$ LOH were more likely than those without it to show increased uptake of ${ }^{201} \mathrm{Tl}$ and, with a weaker association, to be hypermetabolic for ${ }^{18} \mathrm{FDG}$. They reported that elevated metabolism was associated with not only WHO grade but also $1 \mathrm{p} / 19 \mathrm{q} \mathrm{LOH}$. They also speculated that increased ${ }^{201} \mathrm{Tl}$ uptake was associated with angiogenesis in gliomas. These data indicate that oligodendroglial tumors with $1 \mathrm{p} / 19 \mathrm{q} \mathrm{LOH}$ demonstrate increased blood volume due to their higher metabolic demands.

Several studies reported that increased tumor perfusion on MR imaging was associated with $1 \mathrm{p} / 19 \mathrm{q} \mathrm{LOH}$ in oligodendroglial tumors. ${ }^{4,16}$ Oligodendrogliomas often have an attenuated network of branching capillaries that resemble chicken wire. Therefore, CBV values are often higher for oligodendroglial tumors than for astrocytic tumors. ${ }^{17}$ In addition, Watanabe et $\mathrm{al}^{18}$ showed that the presence of a chicken wire vascular pattern was significantly associated with $1 \mathrm{p} / 19 \mathrm{q} \mathrm{LOH}$ in 19 oligodendroglio-

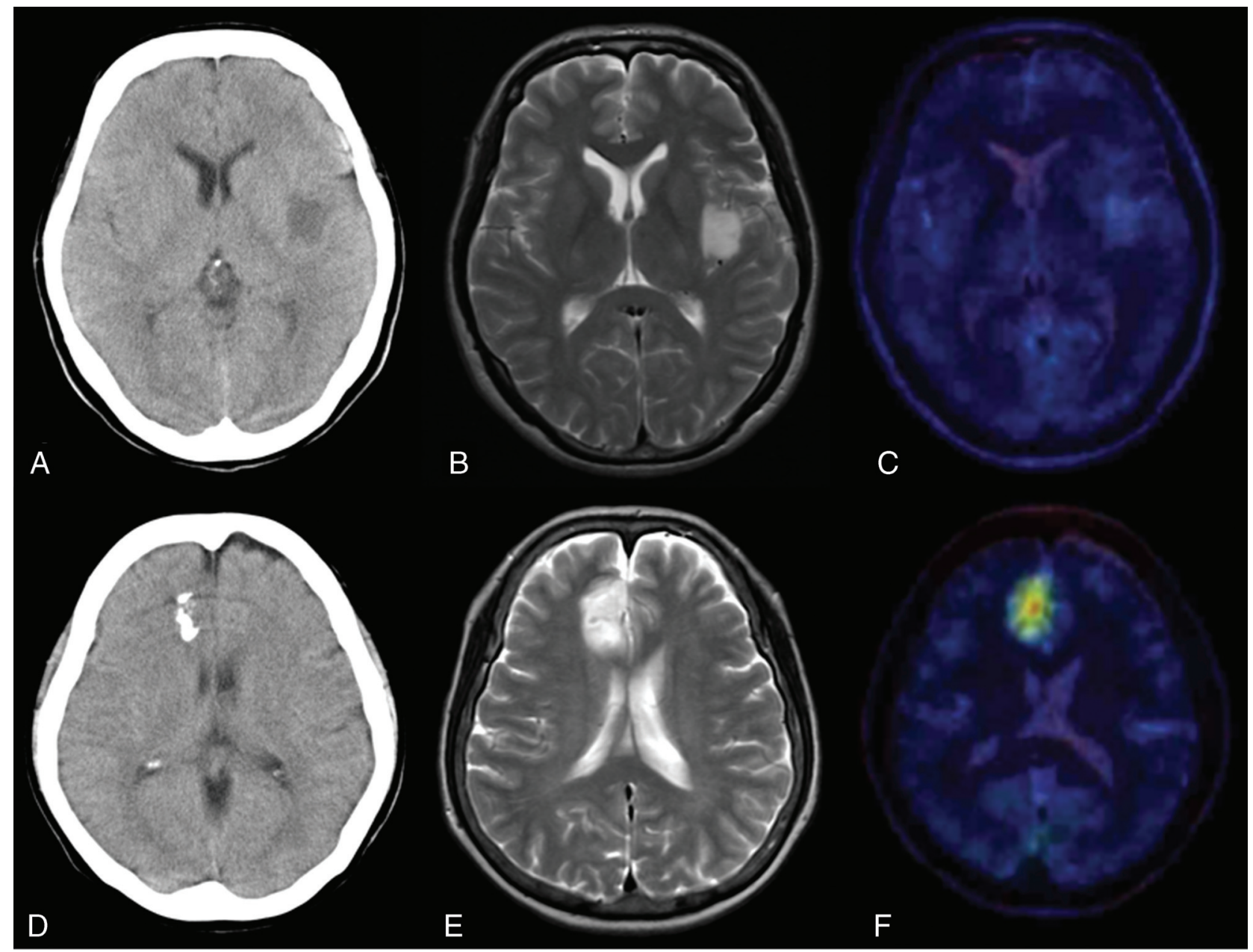

FIG 2. A-C, A representative $1 \mathrm{p} / 19 \mathrm{q} \mathrm{LOH}$-negative oligodendroglioma. $A, \mathrm{CT}$ image shows a left insular tumor without calcification. $B$, T2weighted MR image shows the high-intensity tumor in the insula. C, T2-weighted MR image coregistered with the MET PET image shows the T/N ratio value to be 1.47. $D-F, A$ representative $1 \mathrm{p} / 19 \mathrm{q} \mathrm{LOH}$-positive oligoastrocytoma. $D$, CT scan shows a right frontal tumor with calcification. $E$, T2-weighted MR image shows the high-intensity tumor in the right frontal lobe. F, T2-weighted MR image coregistered with the MET PET image shows the $\mathrm{T} / \mathrm{N}$ ratio value to be 3.66 


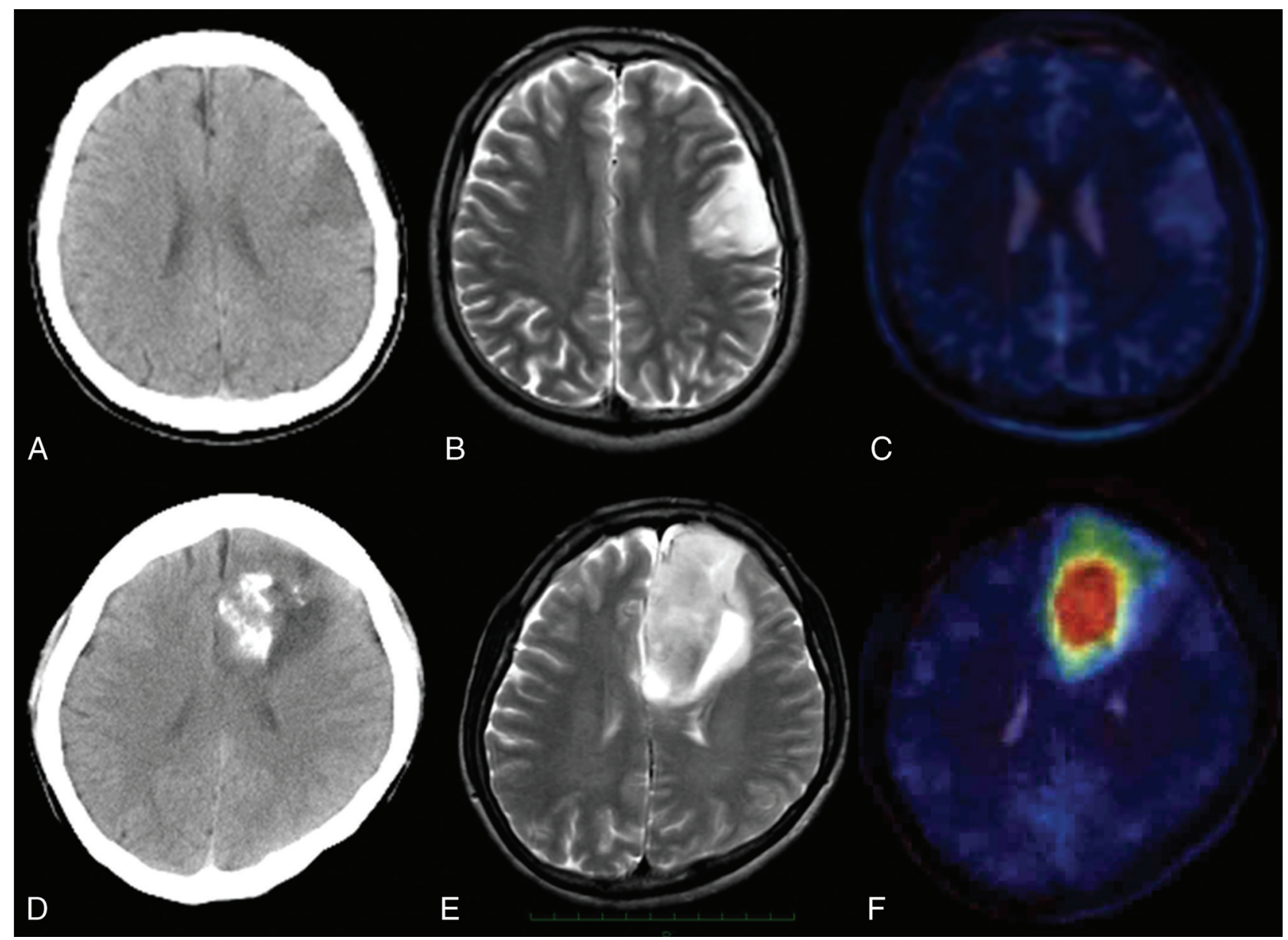

FIG 3. $A-C, A$ representative $1 p / 19 q \mathrm{LOH}$-negative anaplastic oligodendroglioma. $A, C T$ scan shows a left frontal tumor without calcification. $B, T 2$-weighted MR image shows the high-intensity tumor in the left frontal lobe. $C$, T2-weighted MR image coegistered with the MET PET image shows the T/N ratio value to be 1.39. $D-F, A$ representative $1 p / 19 q \mathrm{LOH}-$ positive anaplastic oligoastrocytoma. $D$, CT scan shows a left frontal tumor with calcification. E, T2-weighted MR image shows the high-intensity tumor in the left frontal lobe. $F$, T2-weighted MR image coregistered with the MET PET image shows the T/N ratio value to be 5.94 .

mas. These data also suggest that oligodendroglial tumors with 1p/19q LOH show an increase in tumor blood volume on perfusion MR imaging.

MET PET studies have suggested that MET uptake reflects both angiogenesis ${ }^{8}$ and proliferative activity. ${ }^{7}$ Kato et al $^{7}$ previously reported that the MET T/N ratio did not correlate with the MIB1 labeling index in oligodendroglial tumors. We also found that the $\mathrm{T} / \mathrm{N}$ ratio did not correlate with the MIB1 labeling index in oligodendroglial tumors (data not shown). Nojiri et $\mathrm{al}^{19}$ reported that the increase in tumor microvessel area without an accompanying increase in the microvessel count corresponded to the higher MET uptake in gliomas. Furthermore, Sadeghi et $\mathrm{al}^{20}$ identified a positive correlation between CBV obtained by using perfusion MR images and MET uptake, concluding that the tumor vascular bed represented by CBV plays an important role in MET PET uptake in gliomas. Okita et $\mathrm{al}^{21}$ reported that microvessel density plays much less a part in MET uptake than cell density and the Ki-67 index; however, their study included only 2 oligodendroglial tumors (2/11 patients), which caused results different from ours. Recently, Okubo et $\mathrm{al}^{22}$ demonstrated that expression of LAT1 was significantly correlated with MET uptake in gliomas. Their results indicated that MET transport may be increased by an increased number of microvessels combined with a higher attenuation or activity of LAT1 in the tumor endothelial cells. Consequently, these data suggest that oligodendroglial tumors with $1 \mathrm{p} /$ $19 \mathrm{q}$ LOH show high MET uptake, reflecting not only increased tumor blood volume but also increased transport of MET from the tumor endothelial cells, suggesting a higher metabolic demand. Analyses of these factors add new information on the biology and imaging characteristics of oligodendroglial tumors with 1p/19q LOH.

Our data showed that $\mathrm{T} / \mathrm{N}$ ratios of $1 \mathrm{p} / 19 \mathrm{q} \mathrm{LOH}$-positive tumors were significantly higher than -negative tumors within grade II oligodendroglial tumors. However, $\mathrm{T} / \mathrm{N}$ ratios did not correlate significantly with $1 \mathrm{p} / 19 \mathrm{q} \mathrm{LOH}$ within grade III tumors. Similarly, Kapoor et $\mathrm{al}^{23}$ reported that increased tumor blood volume on perfusion MR imaging studies is associated with $1 \mathrm{p} /$ $19 \mathrm{q} \mathrm{LOH}$ in grade II oligodendrogliomas, but not in grade III tumors. These data suggest that other factors in addition to $1 \mathrm{p} /$ $19 \mathrm{q}$ LOH may contribute to the increase in tumor blood volume, especially within grade III tumors. For example, vascular endothelial growth factor is a marker of angiogenesis and has been shown to correlate with higher grade oligodendroglial tumors, ${ }^{24}$ reflecting endothelial hyperplasia and microvascular prolifera- 
tion. Therefore, we speculate that the $\mathrm{T} / \mathrm{N}$ ratio of grade III oligodendroglial tumors is influenced not only by $1 \mathrm{p} / 19 \mathrm{q} \mathrm{LOH}$ but also by further angiogenesis with increased malignancy.

We demonstrated that $1 \mathrm{p} / 19 \mathrm{q}$ LOH correlated with high MET uptake. While De Witte et $\mathrm{al}^{25}$ reported that high MET uptake represents a poor prognostic factor for WHO grade II and III tumors, they included astrocytic tumors, 31/56 cases, in their study. Oligodendroglial tumors have different mechanisms of MET uptake from astrocytic tumors, reflecting the finding that a tumor vascular bed strongly influences the high MET uptake. Therefore, these tumors should be analyzed separately. Jenkinson et $\mathrm{al}^{4}$ reported that unlike astrocytic gliomas, high CBV values in oligodendroglial neoplasms with 1p/19q LOH do not necessarily indicate aggressive biology, suggestive of differences in the baseline biology of 1p/19q LOH-positive and -negative subtypes. In addition, they speculated that increased vascularity and concomitant drug delivery may contribute to the chemoresponsiveness of such tumors. Therefore, these studies suggest that survival is longer for oligodendroglial tumors with high MET uptake, which is associated with treatment sensitivity.

Our data demonstrated that $1 \mathrm{p} / 19 \mathrm{q}$ LOH status significantly correlated with calcification by using univariate analysis. Calcification is a common radiologic finding in oligodendroglial tumors. Megyesi et $\mathrm{al}^{26}$ reported that 17 of 19 tumors seen to be calcified on CT had 1p/19q LOH. They speculated that calcification is a biologic effect downstream of $1 \mathrm{p} / 19 \mathrm{q}$ LOH. However, their study and ours do not provide definitive evidence for this correlation, and additional study is recommended.

Our study has some limitations. First, we observed no direct correlation between the focus of the MET high uptake and the histopathology. Further investigations, aiming to directly correlate imaging with the histologic findings, such as stereotactic techniques, will strengthen the validity of MET PET as a noninvasive imaging marker of $1 \mathrm{p} / 19 \mathrm{q}$ LOH. Second, our study was retrospective and did not include prognostic information. Therefore, longer and prospective analyses including prognostic values are needed. Third, our data have overlap in T/ $\mathrm{N}$ ratio values between $1 \mathrm{p} / 19 \mathrm{q} \mathrm{LOH}$-positive and -negative tumors. Therefore, the clinical utility of this method should be carefully evaluated and may need an additional adjunctive method to separate $1 \mathrm{p} / 19 \mathrm{q} \mathrm{LOH}$-positive from $\mathrm{LOH}$-negative tumors more clearly, for example, a dynamic PET study.

\section{CONCLUSIONS}

We have demonstrated that high MET uptake is associated with $1 \mathrm{p} / 19 \mathrm{q} \mathrm{LOH}$ in oligodendroglial tumors and that a T/N ratio might be a useful aid in the diagnosis of $1 \mathrm{p} / 19 \mathrm{q}$ LOH. Our data add new information on the biology and imaging characteristics of oligodendroglial tumors with 1p/19q LOH. Further study, including prediction of prognosis, is essential to fully evaluate the role of MET PET in the noninvasive diagnosis of $1 \mathrm{p} / 19 \mathrm{q} \mathrm{LOH}$ in oligodendroglial tumors.

\section{ACKNOWLEDGMENTS}

We thank Takashi Sakayori and Asuka Komori, Department of Pathology, Tokyo Women's Medical University, Tokyo, Japan, for the immunohistochemical staining and the molecular genetic analyses of 1p and 19q losses; and Soko Ikuta, Faculty of Advanced
Techno-Surgery, Institute of Advanced Biomedical Engineering and Science, Tokyo Women's Medical University, for the data analyses of MET PET.

\section{REFERENCES}

1. Reifenberger J, Reifenberger G, Liu L, et al. Molecular genetic analysis of oligodendroglial tumors shows preferential allelic deletions on 19q and 1p. Am J Pathol 1994;145:1175-90

2. Cairncross G, Jenkins R. Gliomas with $1 \mathbf{p} / 19 \mathbf{q}$ codeletion: a.k.a. oligodendroglioma. Cancer J 2008;14:352-57

3. Bourne TD, Schiff D. Update on molecular findings, management and outcome in low-grade gliomas. Nat Rev Neurol 2010;6:695-701

4. Jenkinson MD, Smith TS, Joyce KA, et al. Cerebral blood volume, genotype and chemosensitivity in oligodendroglial tumours. $\mathrm{Neu}$ roradiology 2006;48:703-13

5. McGirt MJ, Chaichana KL, Attenello FJ, et al. Extent of surgical resection is independently associated with survival in patients with hemispheric infiltrating low-grade gliomas. Neurosurgery 2008;63: 700-07, author reply 707-08

6. Ogawa T, Inugami A, Hatazawa J, et al. Clinical positron emission tomography for brain tumors: comparison of fludeoxyglucose $\mathrm{F} 18$ and L-methyl-11C-methionine. AJNR Am J Neuroradiol 1996;17:345-53

7. Kato T, Shinoda J, Oka N, et al. Analysis of ${ }^{11} \mathrm{C}$-methionine uptake in low-grade gliomas and correlation with proliferative activity. AJNR Am J Neuroradiol 2008;29:1867-71

8. Kracht LW, Friese M, Herholz K, et al. Methyl-[11C]-l-methionine uptake as measured by positron emission tomography correlates to microvessel density in patients with glioma. Eur J Nucl Med Mol Imaging 2003;30:868-73

9. Maia AC Jr, Malheiros SM, da Rocha AJ, et al. MR cerebral blood volume maps correlated with vascular endothelial growth factor expression and tumor grade in nonenhancing gliomas. AJNR Am J Neuroradiol 2005;26:777-83

10. Akobeng AK. Understanding diagnostic tests 3: receiver operating characteristic curves. Acta Paediatr 2007;96:644-47

11. Smith JS, Perry A, Borell TJ, et al. Alterations of chromosome arms $1 p$ and $19 q$ as predictors of survival in oligodendrogliomas, astrocytomas, and mixed oligoastrocytomas. J Clin Oncol 2000;18: $636-45$

12. van den Bent MJ, Carpentier AF, Brandes AA, et al. Adjuvant procarbazine, lomustine, and vincristine improves progression-free survival but not overall survival in newly diagnosed anaplastic oligodendrogliomas and oligoastrocytomas: a randomized European Organisation for Research and Treatment of Cancer phase III trial. J Clin Oncol 2006;24:2715-22

13. Jenkinson MD, du Plessis DG, Smith TS, et al. Histological growth patterns and genotype in oligodendroglial tumours: correlation with MRI features. Brain 2006;129:1884-91

14. Brown R, Zlatescu M, Sijben A, et al. The use of magnetic resonance imaging to noninvasively detect genetic signatures in oligodendroglioma. Clin Cancer Res 2008;14:2357-62

15. Walker C, du Plessis DG, Fildes D, et al. Correlation of molecular genetics with molecular and morphological imaging in gliomas with an oligodendroglial component. Clin Cancer Res 2004;10: 7182-91

16. Whitmore RG, Krejza J, Kapoor GS, et al. Prediction of oligodendroglial tumor subtype and grade using perfusion weighted magnetic resonance imaging. J Neurosurg 2007;107:600-09

17. Lev MH, Ozsunar Y, Henson JW, et al. Glial tumor grading and outcome prediction using dynamic spin-echo MR susceptibility mapping compared with conventional contrast-enhanced MR: confounding effect of elevated rCBV of oligodendrogliomas [corrected]. AJNR Am J Neuroradiol 2004;25:214-21

18. Watanabe T, Nakamura M, Kros JM, et al. Phenotype versus genotype correlation in oligodendrogliomas and low-grade diffuse astrocytomas. Acta Neuropathol 2002;103:267-75 
19. Nojiri T, Nariai T, Aoyagi M, et al. Contributions of biological tumor parameters to the incorporation rate of L: -[methyl-(11)C] methionine into astrocytomas and oligodendrogliomas. J Neurooncol 2009;93:233-41

20. Sadeghi N, Salmon I, Decaestecker C, et al. Stereotactic comparison among cerebral blood volume, methionine uptake, and histopathology in brain glioma. AJNR Am J Neuroradiol 2007;28:455-61

21. Okita Y, Kinoshita M, Goto T, et al. (11)C-methionine uptake correlates with tumor cell density rather than with microvessel density in glioma: a stereotactic image-histology comparison. Neuroimage 2010;49:2977-82

22. Okubo S, Zhen HN, Kawai N, et al. Correlation of L-methyl-11Cmethionine (MET) uptake with L-type amino acid transporter 1 in human gliomas. J Neurooncol 2010;99:217-25
23. Kapoor GS, Gocke TA, Chawla S, et al. Magnetic resonance perfusion-weighted imaging defines angiogenic subtypes of oligodendroglioma according to $1 \mathbf{p} 19 \mathbf{q}$ and EGFR status. J Neurooncol 2009;92:373-86

24. Christov C, Adle-Biassette H, Le Guerinel C, et al. Immunohistochemical detection of vascular endothelial growth factor (VEGF) in the vasculature of oligodendrogliomas. Neuropathol Appl Neurobiol 1998;24:29-35

25. De Witte O, Goldberg I, Wikler D, et al. Positron emission tomography with injection of methionine as a prognostic factor in glioma. J Neurosurg 2001;95:746-50

26. Megyesi JF, Kachur E, Lee DH, et al. Imaging correlates of molecular signatures in oligodendrogliomas. Clin Cancer Res 2004;10: 4303-06 